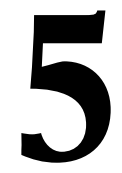

\title{
PERCEPCIÓN SOBRE EL PLAGIO ACADÉMICO DE ESTUDIANTES UNIVERSITARIOS ESPAÑOLES
}

\author{
(PERCEPTION OF ACADEMIC PLAGIARISM BY SPANISH UNIVERSITY \\ STUDENTS)
}

Violeta Cebrián-Robles

Manuela Raposo-Rivas

Universidad de Vigo

Manuel Cebrián-de-la-Serna

Universidad de Málaga

José Antonio Sarmiento-Campos

Universidad de Vigo

DOI: $10.5944 / e d u c X X 1.20062$

Cómo referenciar este artículo/How to reference this article:

Cebrián-Robles, V.; Raposo-Rivas, M.; Cebrián-de-la-Serna, M. y Sarmiento-Campos, J.A. (2018). Percepción sobre el plagio académico de estudiantes universitarios españoles. Educación XX1, 21(2), 105-129, doi: 10.5944/educXX1.20062

Cebrián-Robles, V.; Raposo-Rivas, M.; Cebrián-de-la-Serna, M. \& Sarmiento-Campos, J.A. (2018) Percepción sobre el plagio académico de estudiantes universitarios españoles. [Perception of academic plagiarism by spanish university students]. Educación XX1, 21(2), 105-129, doi: 10.5944/educXX1.20062

\section{RESUMEN}

Los procesos de enseñanza y aprendizaje cada vez más digitalizados han convertido Internet en la principal fuente de información en las universidades. La facilidad para el acceso a la información puede ir acompañada de prácticas fraudulentas como el plagio. Por ello, la finalidad del estudio es conocer el origen de las causas y motivaciones que poseen los estudiantes universitarios españoles; así como establecer un modelo que permita categorizarlas y que aporte explicación teórica a los datos empíricos. Con un diseño de investigación correlacional causal y utilizando como instrumento de medida un cuestionario ad hoc, se indaga sobre qué prácticas considera que son deshonestas, si ha realizado alguna vez estas prácticas y el motivo principal. Participan 461 estudiantes universitarios de Educación (grado y máster) pertenecientes a 
cinco universidades españolas. A partir del análisis factorial exploratorio se identifica un factor relativo al concepto de plagio, dos sobre ¿qué hacen? y tres factores en ¿por qué lo hacen? Con ello, se realiza un análisis factorial confirmatorio del modelo empírico basado en el tipo de relación que, según el alumnado, se produce entre el concepto de plagio y sus elementos constitutivos, las acciones consideradas plagio que realizan y los diversos motivos para llevarlas a cabo. Se obtiene un modelo ajustado según los índices estadísticos: Chi cuadrado normalizado $(1,9)$, bondad del ajuste $(0,97)$, bondad del ajuste corregido $(0,97)$, ajuste comparativo $(0,95)$, error de aproximación cuadrático medio $(0,045)$ y criterio informativo de Akaike (mucho más bajo que el del modelo independiente). Se concluye identificando un patrón en el que a pesar de conocer lo que es el "plagio" y sus consecuencias, se justifican tres motivos interrelacionados para plagiar: «internos» a la persona, «externos» a ella y la falta de motivación e interés por la tarea planteada.

\title{
PALABRAS CLAVE
}

Derechos de autor; plagio; competencia ética; profesional de la educación; honestidad académica.

\begin{abstract}
Increasingly digitalized teaching and learning processes have turned the Internet into the main source of information in universities. The ease of access to information can be accompanied by fraudulent practices such as plagiarism. Therefore, the purpose of this study is to discover the origin of the reasons and motivations that Spanish university students have, as well as to establish a model that allows them to be categorized and that provides a theoretical explanation for the empirical data. With a causal correlational research design and using an ad hoc questionnaire as an instrument of measurement, to investigate which practices they consider to be dishonest, if they have ever carried out these practices and the main reason for this. 461 university students in Education (degree and master's degree) at five Spanish universities participated in the study. From the exploratory factor analysis, one factor is identified relative to the concept of plagiarism, two about what they do, and three factors about why they do it. With this, a confirmatory factor analysis is performed on the empirical model based on the type of relationship that, according to the students, occurs between the concept of plagiarism and its constituent elements, the actions considered plagiarism carried out by these students and the various reasons for carrying them out. A model is obtained according to the statistical indices: standardized Chi square (1.9), goodness of fit (0.97), goodness of corrected fit (0.97), comparative fit (0.95), mean quadratic approximation error (0.04)
\end{abstract}


VIOLETA CEBRIÁN-ROBLES, MANUELA RAPOSO-RIVAS, MANUEL CEBRIÁN-DE-LA-SERNA, JOSÉ ANTONIO SARMIENTO-CAMPOS

PERCEPCIÓN SOBRE EL PLAGIO ACADÉMICO DE ESTUDIANTES UNIVERSITARIOS ESPAÑOLES

and Akaike's informative criterion, which is lower than the independent model. It concludes by identifying a pattern in which, despite knowing what "plagiarism» is and its consequences, three interrelated reasons are justified for plagiarism: «internal» to the person, «external» to them and lack of motivation and interest in the task.

\section{KEYWORDS}

Copyright; plagiarism; ethic competence; professional education; academic honesty.

\section{INTRODUCCIÓN}

El acelerado desarrollo tecnológico de Internet sitúa a todas las instituciones universitarias del mundo en una labor permanente para desarrollar, experimentar y utilizar estas tecnologías con integridad académica (Bretag, 2016), máxime cuando se han convertido en la principal fuente de búsqueda de documentación académica para los estudiantes universitarios (Sanvicén \& Molina, 2015), pero también en una fuente de plagio (Caldevilla, 2010).

Si bien hay un acuerdo generalizado sobre la importancia de este problema, resulta paradójico que aún no tengamos un corpus práctico que acoja una definición consensuada, ni un acuerdo sobre cómo prevenirlo. En la revisión de numerosos estudios se comprueba que la definición de plagio viene precedida de una aclaración sobre la dificultad que ello conlleva, que deriva al final en una falta de consenso (Adam, Anderson, \& SpronkenSmith, 2017; Ruipérez \& García-Cabrero, 2016). Por esta razón, partimos de la definición que del verbo plagiar ofrece la Real Academia Española (2001): copiar en lo sustancial obras ajenas, dándolas como propias. Por su parte, Kolich (1983 cit. en Zarfsaz \& Ahmadi, 2017) relaciona el plagio con la integridad académica, y esto es sinónimo de confianza, imparcialidad, respeto y responsabilidad. Igualmente, según la teoría de la «decisión racional» (Sattler, Graeff, \& Willem, 2013), el plagio es una "conducta desviada» donde existe una relación entre la utilidad y las oportunidades.

La vulneración de los derechos de autor por parte de los estudiantes es, entre otros muchos, un tema recurrente a nivel internacional (Park, 2003; Do Ba et al., 2017; Khoshsaligheh, Mehdizadkhani, \& Keyvan, 2017). Las investigaciones más relevantes se centran, por un lado, en comprender cómo entienden los estudiantes las normativas que se articulan en las universidades, y qué percepción tienen sobre el derecho de autor; y por 
otro, se preguntan qué papel representan los docentes y gestores sobre las políticas y estrategias a seguir para su prevención (Adam, 2016). Existen otros trabajos que pretenden conocer en profundidad qué piensan, qué mueve a los estudiantes y qué acciones proactivas son más eficaces (Amiri \& Razmjoo, 2016; Adam, Anderson, \& Spronken-Smith, 2017). Al mismo tiempo que se identifican estudios que abarcan diferentes áreas y contextos geográficos (Hu \& Sun, 2017; Kokkinaki, Demoliou \& Iakovidou, 2015), así como estudios comparados entre países y culturas diferentes (Cosma et al., 2017; Guerrero, Mercado \& Marina, 2017; Ehrich et al., 2016).

En España e Iberoamérica esta temática es de reciente actualidad, identificándose un punto de inflexión importante en la década pasada con los trabajos de Comas-Forgas, \& Sureda-Negre (2007), Sureda-Negre, Comas-Forgas, \& Morey (2009); incrementando su productividad en esta (Montecinos, 2013; Sureda-Negre, Comas-Forgas \& Oliver-Trobat, 2015; Cebrián-Robles, Raposo-Rivas, \& Sarmiento-Campos, 2016; Ochoa \& Cueva, 2016).

Un número importante de investigaciones confluyen al tratar de conocer el motivo que provoca estas prácticas deshonestas, diferenciándose la óptica de estudio (Balbuena \& Lamela, 2015): la facilidad de las tecnologías (Ennam, 2017), la perspectiva de los profesores (Sureda et al., 2009), las diferencias étnicas y culturales (Hayes \& Introna, 2005; Tayan, 2016) y aquellos que comparan dentro de una misma institución estudiantes de diferentes áreas (Comas-Forgas, \& Sureda-Negre, 2010). Por su parte, Guerrero, Marina \& Mercado (2016, pp. 13), aseguran que un ambiente institucional de incertidumbre, con reglas poco claras, solo obliga a los estudiantes a multiplicar sus esfuerzos para realizar publicaciones, lo que "podría incidir en la generación de estrés laboral y constituir un ambiente favorable para la comisión de conductas no éticas". Estos hallazgos coinciden con los de Do Ba, et. al., (2017) quienes establecen una correlación negativa entre rendimiento académico y las inclinaciones a copiar y pegar; al mismo tiempo que hallaron un impacto positivo cuando se plantean las políticas disuasorias y el uso de plataformas antiplagio.

Se ha discutido también sobre la efectividad de las herramientas de detección de plagio. Para Youmans, (2011) el plagio correlaciona inversamente con la advertencia o el conocimiento de estas herramientas. Si bien, es obvio considerar que no basta solo con la tecnología, necesitamos plantear una perspectiva más amplia con políticas de prevención, con las tecnologías antiplagio como variable dependiente (Heckler, Forde, \& Bryan, 2013) y como variable independiente (Kokkinaki, Demoliou, \& Iakovidou, 2015). 
VIOLETA CEBRIÁN-ROBLES, MANUELA RAPOSO-RIVAS, MANUEL CEBRIÁN-DE-LA-SERNA, JOSÉ ANTONIO SARMIENTO-CAMPOS

PERCEPCIÓN SOBRE EL PLAGIO ACADÉMICO DE ESTUDIANTES UNIVERSITARIOS ESPAÑOLES

Por otro lado, el estudio de Kuntz \& Butler (2014) indica que los principales predictores de las actitudes de los estudiantes en la aceptación del plagio se deben a factores individuales (el género y la personalidad) y a la sensibilidad por la justicia. Siendo la comprensión de las normas universitarias sobre el plagio el mayor predictor, más que las diferencias debidas a las disciplinas de los estudiantes y la percepción que del plagio muestran estudiantes y docentes, estando a su vez muy relacionada con el conocimiento y confusión que se tiene al respecto.

Cada vez son más las investigaciones que tratan de encontrar cuáles son las mejores técnicas de prevención del plagio (Sattler, Wiegel \& Veen, 2017; Chankova, 2017), donde la solución a la vulneración de los derechos de autor por parte de los estudiantes pasa por eliminar el carácter punitivo. Este es el caso de Eaton, Guglielmin \& Otoo (2017) quienes abordan el problema desde una perspectiva formativa sobre integridad académica, recomendando cuatro líneas de acción para los educadores: a) llevar a cabo conversaciones sobre plagio, b) enseñar a los estudiantes las habilidades para citar y referenciar, c) crear oportunidades de retroalimentación como parte del proceso de aprendizaje y, d) llevar a cabo evaluaciones para incluir retroalimentación formativa y sumativa.

Por ello, los docentes pueden hacer mucho en su labor formativa, desde el diseño de los cursos, pasando por las tutorías, hasta llegar a la supervisión de los trabajos. Como demuestran Heckler, Forde, \& Bryan (2013) al comprobar que, tareas como el diseño de ejercicios de escritura, pueden mitigar el plagio. Esto se producía cuando en las tareas se solicitaba a los estudiantes justificación de fuentes para sus argumentos y citaciones adecuadas de las publicaciones utilizadas en Internet. Similares resultados obtienen McGowan \& Lightbody, (2008) cuando introdujeron en los ejercicios de estudiantes de económicas la auto-revisión y revisiones de pares sobre el plagio. No hay dudas, por tanto, en la necesidad de plantear medidas formativas y de supervisión, donde predomine tanto el diálogo reflexivo entre docente y estudiantes, como los ejercicios de autorreflexión (López, 2014; Dalal, 2015).

Finalmente, hay consenso en la literatura en cuanto a que las medidas coercitivas y punitivas no son exitosas (Eaton, Guglielmin \& Otoo, 2017); entre otras, porque si cambian las prácticas por este motivo, cuando cambie a un contexto más permisivo, estas prácticas se repetirán (Dalal, 2015). Por lo que sería recomendable atajar las concepciones que llevan a los usuarios a tales conductas, sin olvidar otros motivos ya señalados (mala planificación en las tareas y entrega de evidencias, formación para citar correctamente, etc.). Para acercarnos a este conocimiento es interesante averiguar si hay un modelo común en los estudiantes que esté relacionado con contextos 
físicos o culturales bien definidos, como puede ser una titulación, facultad, universidad, una región, un país, etc.

La investigación que se presenta se sitúa dentro de este último enfoque, forma parte de un trabajo más amplio sobre la evaluación de los aprendizajes (1), y plantea en este trabajo, el siguiente objetivo:

"Aproximación al conocimiento y actuaciones sobre el plagio de los estudiantes universitarios de titulaciones de Educación, para identificar un patrón que categorice sus causas y motivaciones.»

\section{MÉTODO}

La investigación desarrollada responde, desde la óptica de Hernández, Fernández y Baptista (2014), a un diseño no experimental de tipo transversal, con una línea secuencial que parte de una intención inicial de tipo exploratoriodescriptivo y que, a partir del conocimiento adquirido en este primer momento, evoluciona a un enfoque de tipo explicativo. Atendiendo a las técnicas empleadas, incluimos el estudio dentro de los diseños correlacionales causales, pues la utilización del análisis factorial exploratorio cubriría el campo correlacional, mientras que el análisis factorial confirmatorio haría lo mismo con el causal (Batista \& Coenders, 2000; Kerlinger \& Lee, 2002).

El proyecto ha centrado la atención en estudiantes de titulaciones de educación de cinco universidades españolas, para preguntarles: ¿cuáles de las siguientes prácticas consideras que son deshonestas o son motivo de plagio?, ¿has realizado alguna vez alguna de estas prácticas?, y en caso de haber realizado alguna de las prácticas anteriores ¿cuál fue tu motivo principal?

Por lo tanto, cabría plantear como pregunta de investigación: ¿existe un modelo o patrón subyacente en los estudiantes de educación en España que muestre la relación existente entre las causas del plagio?

\section{Participantes}

Teniendo en cuenta que lo que se investiga es la presencia e identificación de algún modelo global sobre plagio, que aporte una explicación teórica a los datos empíricos, se hizo una selección de participantes estratégica e intencional (Perelló, 2009: 27). En dicha selección se procuró la representatividad poblacional, en cuanto a tipo de estudio (grado/máster) y titulación de Educación. Con respecto a la suficiencia, 
VIOLETA CEBRIÁN-ROBLES, MANUELA RAPOSO-RIVAS, MANUEL CEBRIÁN-DE-LA-SERNA, JOSÉ ANTONIO SARMIENTO-CAMPOS

PERCEPCIÓN SOBRE EL PLAGIO ACADÉMICO DE ESTUDIANTES UNIVERSITARIOS ESPAÑOLES

teniendo en cuenta el número de posibles informantes y la dificultad de acceder a un número estadísticamente significativo utilizando métodos clásicos, más recomendados para estudios comparativos y generalistas, se han utilizado técnicas de bootstrap (Efron \& Tibshirani, 1994) como método de remuestreo en el proceso de búsqueda del modelo, para así poder minimizar los posibles efectos adversos derivados del tipo de muestreo utilizado y aportar robustez a los resultados.

Dicha selección atiende a dos criterios: a) pertenecientes a cinco universidades españolas (Barcelona, Granada, Málaga, León y Vigo) miembros del proyecto $\mathrm{I}+\mathrm{D}+\mathrm{i}(1)$, b) estudiantes del ámbito de educación (grado y máster). Participaron 461 estudiantes, $348(75,5 \%)$ alumnas y 113 alumnos $(24,5 \%)$. De ellos, $209(45,3 \%)$ estudian el grado de educación primaria, $95(20,6 \%)$ pedagogía, $86(18,7 \%)$ educación infantil, 30 (6,5\%) otros másteres relacionados con el campo educativo, 21 (4,6\%) el máster de secundaria y $20(4,3 \%)$ educación social. Se da una distribución homogénea por cursos académicos, de $1 .^{\circ}$ a $4 .^{\circ}$ en el grado junto con el postgrado, en torno al 20\%. Por lo que respecta a sus edades, oscilan entre los 18 y 25 años. Se puede observar cierta falta de representatividad geográfica aunque, este hecho y las variables asociadas al mismo, no nos parecen determinantes en los resultados.

\section{Instrumento}

A partir de instrumentos ya validados (Comas-Forgas \& SuredaNegre, 2010; Sureda-Negre, Comas-Forgas, \& Oliver-Trobat, 2015; Ehrich et al., 2016) se elaboró un cuestionario on-line mediante la herramienta Limeservey. Tras una aplicación piloto y revisión de expertos, quedó constituido por cuatro bloques: datos de contextualización (universidad, género, edad, titulación y curso); el plagio y sus motivaciones (5 preguntas con un total de 53 ítems); soluciones para evitar el plagio (5 preguntas con un total de 26 ítems), medidas a tomar en caso de plagio (1 pregunta con 6 ítems) y formación específica sobre la temática (1 pregunta).

Si consideramos los elementos que constituyen las tres cuestiones del segundo bloque analizadas en este trabajo, sobre el concepto ( $¿$ cuáles de las siguientes prácticas consideras que son deshonestas o son motivo de plagio?), la acción (¿has realizado alguna vez alguna de estas prácticas?), y las motivaciones (en caso de haber realizado alguna de las prácticas anteriores ¿cuál fue tu motivo principal?), el estadístico alpha de Cronbach es de 0,$843 ; 0,7$ y 0,922 respectivamente. El alpha del cuestionario total es de 0,881 . Todos ellos índices suficientes para este tipo de estudio (Huh, Delorme \& Reid, 2006). 


\section{PROCEDIMIENTO Y ANÁLISIS DE LOS DATOS}

Para obtener los resultados del análisis factorial exploratorio (AFE) empleando Bootstrap, así como los derivados del análisis de clúster, se ha utilizado el paquete estadístico PSYCH en R (Revelle, 2017), que incluye las funciones actualizadas más empleadas en la investigación psicológica y de la personalidad. Para la toma de decisiones que derivan del AFE seguimos las indicaciones de Lloret-Segura et al. (2014), más específicamente en aquellas cuestiones sobre la adecuación del análisis factorial confirmatorio (AFC), idoneidad de la muestra, método de estimación de factores y número de factores a retener. Para establecer el número de iteraciones más idóneo hemos seguido a Díaz-Emparanza (1996) siendo 1.000 repeticiones suficientes para los objetivos propuestos.

La figura 1 muestra el proceso seguido para realizar el análisis de los datos obtenidos. Como se pretende llegar a un modelo empírico con justificación teórica ajustado mediante AFC que conserve la mayor parte de información recogida a través de los ítems que conforman las tres cuestiones estudiadas, se define un paso intermedio que identifica factores subyacentes mediante la simultaneidad y verificación de ajuste de dos técnicas, como son el AFE (análisis factorial exploratorio) y el análisis jerárquico de conglomerados. Para la especificación y posterior verificación y ajuste del modelo, se emplean Ecuaciones estructurales.

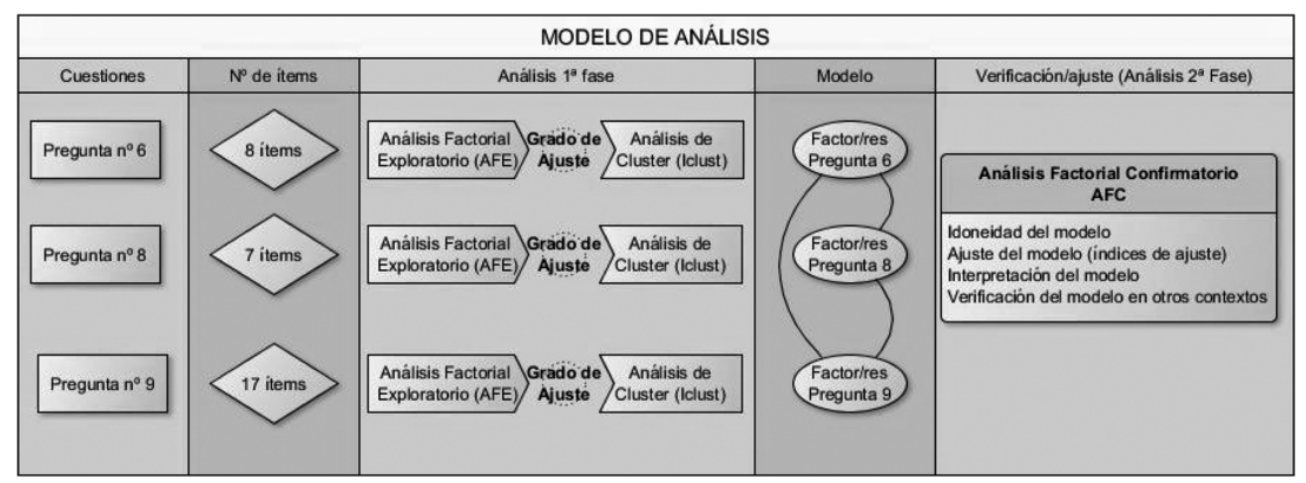

Figura 1. Diseño del proceso de análisis de los datos

No obstante, esta es una técnica muy exigente en cuanto a la naturaleza y relaciones entre los datos, de ahí que se haya recurrido en una primera fase al AFE que, junto con el análisis de clúster, nos permite reducir los datos e identificar factores más inclusivos, y variables con mayores cargas factoriales que luego se utilizarían en el AFC. Vendría a resultar el proceso que Tirado \& Aguaded (2012) definen como reducción factorial previa y conocido también como método de parcelización (Hall, Snell \& Singer 
VIOLETA CEBRIÁN-ROBLES, MANUELA RAPOSO-RIVAS, MANUEL CEBRIÁN-DE-LA-SERNA, JOSÉ ANTONIO SARMIENTO-CAMPOS

PERCEPCIÓN SOBRE EL PLAGIO ACADÉMICO DE ESTUDIANTES UNIVERSITARIOS ESPAÑOLES

Foust, 1999) para obtener los factores en el AFE y posteriormente para seleccionar las variables exógenas en el AFC. Un problema común en el AFE es determinar el número apropiado de factores a considerar. Hemos optado por tener en cuenta dos criterios: VSS (Very Simple Structure) de Revelle y Rocklin (1979) y el criterio MAP (Minimum Average Partial) de Velicer (1976).

\section{RESULTADOS}

La pregunta 6 indaga sobre el concepto de plagio (¿cuáles de las siguientes prácticas consideras que son deshonestas o son motivo de plagio?) considerando 8 ítems:

- p1. Entregar un trabajo realizado por otro alumno/a que ya había sido entregado en cursos anteriores (para la misma asignatura o para otra).

- p2. Copiar de páginas web fragmentos de textos y - sin citarpegarlos directamente en un documento - en el cual hay una parte de texto escrita por uno mismo- y entregarlo como trabajo de una asignatura.

- p3. Bajar un trabajo completo de Internet y entregarlo, sin modificar, como trabajo propio de una asignatura.

- p4. Copiar fragmentos de fuentes impresas (libros, enciclopedias, periódicos, artículos de revista, etc.) y añadirlos — sin citar- como partes de un trabajo propio de una asignatura.

- p5. Hacer íntegramente un trabajo a partir de fragmentos copiados literalmente de páginas web (sin que ninguna parte del trabajo haya sido realmente escrita por el alumno/a).

- p6. Copiar partes de mis trabajos entregados durante cursos anteriores y usarlos como apartados de un trabajo nuevo.

- p7. Copiar imágenes, vídeos y sonidos de Google sin indicar la autoría y dónde se obtuvo.

- p8. Debido a que el plagio consiste en tomar palabras de otra persona y no sus bienes materiales, el plagio no es gran cosa.

En ella, tanto los criterios VSS como MAP indican que lo apropiado sería considerar solo un factor, que explicaría el 53\% de la varianza total. El valor de RMSR fue de 0.07, el índice de Tucker Lewis (TLI) de 0,814 y el índice KMO de 0,873. En el siguiente gráfico (figura 2) podemos observar las cargas factoriales de cada uno de los ítems y sus intervalos de confidencia Bootstrap. 


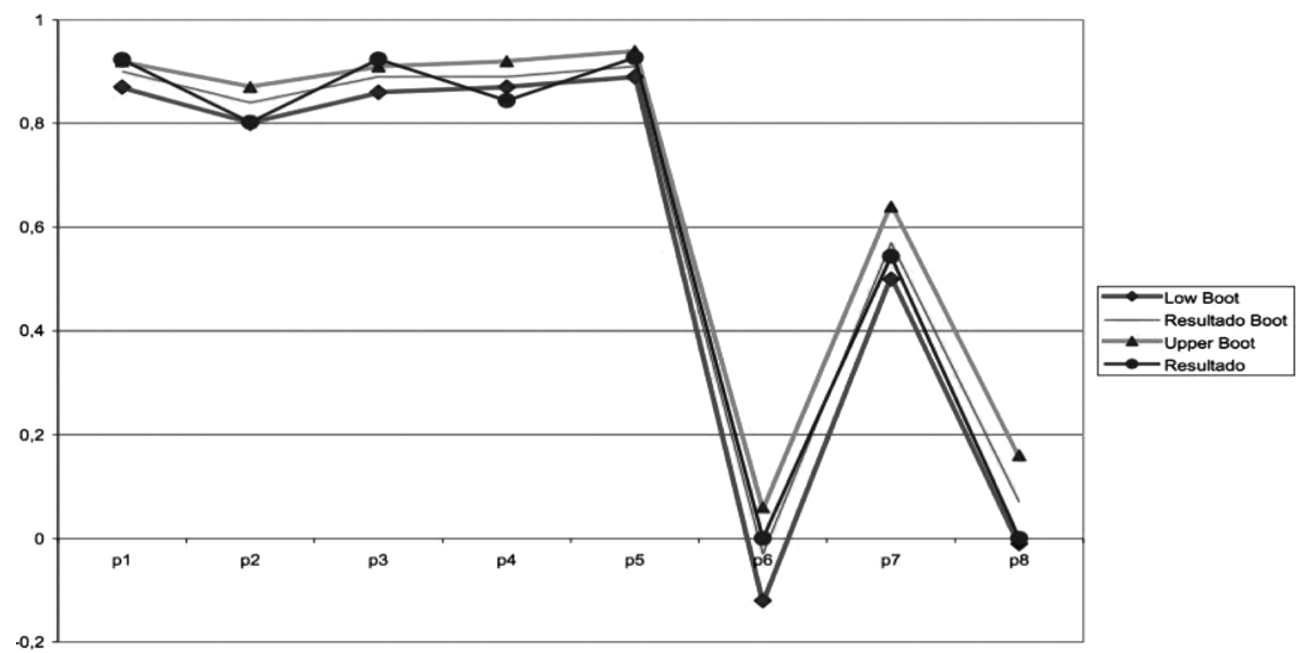

Figura 2. Cargas factoriales resultado del análisis factorial con Bootstrap de los ítems correspondientes al concepto de plagio

La mayor carga factorial aparece en los cinco primeros ítems, lo que significa que en su concepto de plagio los estudiantes no incluyen el autoplagio (p6) ni la apropiación de material audiovisual (p7). Aunque no consideran el plagio como un asunto irrelevante (p8).

La pregunta 8 sobre las acciones que significan plagio ( $¿$ has realizado alguna vez alguna de estas prácticas?) posee las mismas opciones de respuesta que la anterior para diferenciar el concepto de la actuación. En esta, los criterios VSS y MAP coinciden en indicar la idoneidad de considerar dos factores, que explicarían el $40 \%$ de la varianza. $\mathrm{KMO}=0,776$, RMSR $=0,02$ y TLI $=0,977$. Un factor se corresponde con el plagio «parcial» (p2. Copiar de páginas..., p4. Copiar fragmentos..., p7. Copiar imágenes...) y el otro con el plagio «total» (p1. Entregar un trabajo realizado por otro..., p3. Bajar un trabajo completo..., p5. Hacer íntegramente un trabajo...).

Con respecto a las motivaciones — pregunta 9-: en caso de haber realizado alguna de las prácticas anteriores ¿cuál fue tu motivo principal? las opciones de respuesta son las siguientes:

- ítem 1. Porque tuve que redactar un trabajo muy extenso a lo que no estaba acostumbrado.

- ítem 2. Por adquirir una mayor calificación.

- ítem 3. Por desconocer el uso adecuado de citas.

- ítem 4. No sé redactar tanto texto en tan poco tiempo.

- ítem 5. Por desinterés en la tarea. 
VIOLETA CEBRIÁN-ROBLES, MANUELA RAPOSO-RIVAS, MANUEL CEBRIÁN-DE-LA-SERNA, PERCEPCIÓN SOBRE EL PLAGIO ACADÉMICO DE ESTUDIANTES UNIVERSITARIOS ESPAÑOLES

— ítem 6. Escribir sobre algo que desconozco.

- ítem 7. Escribir sobre algo que no comprendo.

- ítem 8. Son muchas tareas, y me organizo mal el tiempo y lo realizo al final.

— ítem 9. Porque no hay peligro, no se toma ninguna acción en los casos de plagio.

- ítem 10. Es difícil averiguar dónde copiaste.

- ítem 11. Desconozco la normativa institucional.

- ítem 12. Porque no se aprendía mucho en la asignatura.

— ítem 13. El profesorado no tiene mucho tiempo para saber si hubo plagio.

— ítem 14. Me sentí tentado porque otros copian y tienen buenas notas.

— ítem 15. El plagio se justifica cuando el profesorado pide demasiadas tareas.

- ítem 16. El profesorado no tiene muchas competencias para detectarlo.

— ítem 17. Nunca hice ninguna de las prácticas anteriores.

Después de su análisis factorial, y considerando los criterios VSS y MAP, seleccionamos 3 factores que explican el 45\% de la varianza. El índice KMO arrojó un valor de 0,933. RMSR de 0,04 y TLI de 0,868.

La función empleada para el análisis de conglomerados aplica el algoritmo iclust para agrupar jerárquicamente los elementos para formar escalas compuestas. Los clústers se forman hasta que el coeficiente $\alpha$ Cronbach o $\beta$ Revelle no aumentan. Ambos coeficientes son estimaciones de la fiabilidad y la saturación general de los factores de la prueba (Revelle, 1979; Revelle, \& Zinbarg, 2009).

El primero de los tres factores identificados sitúa el origen de la motivación para el plagio en causas «externas» (ítems 9, 13 y 16). El segundo, se relaciona con causas «internas» (ítems 1, 6 y 7). El tercer factor agrupa dos ítems en los que aparece el desinterés (ítems 5 y 12).

Seguidamente, se muestra el buen ajuste de los factores extraídos mediante el análisis factorial con los conglomerados resultantes del análisis de clúster, a través del factor de congruencia expresado como el valor del coseno (Tabla 1). 
Tabla 1

Comparación resultados análisis factorial con los conglomerados

\begin{tabular}{|c|c|c|c|c|c|c|c|}
\hline \multirow[b]{2}{*}{ Cuestión } & \multirow[b]{2}{*}{ Factor } & \multicolumn{3}{|c|}{ Valor del coseno } & \multicolumn{3}{|c|}{ Ángulo } \\
\hline & & $\begin{array}{c}\text { Cluster } \\
\text { I }\end{array}$ & $\begin{array}{c}\text { Cluster } \\
\text { II }\end{array}$ & $\begin{array}{c}\text { Cluster } \\
\text { III }\end{array}$ & $\underset{\text { I }}{\text { Cluster }}$ & $\begin{array}{c}\text { Cluster } \\
\text { II }\end{array}$ & $\begin{array}{c}\text { Cluster } \\
\text { III }\end{array}$ \\
\hline Pregunta 6 & I & 1,00 & & & $0^{\circ}$ & & \\
\hline \multirow{2}{*}{ Pregunta 8} & I & 0,94 & 0,75 & & $19^{\circ}$ & $41^{\circ}$ & \\
\hline & II & 0,48 & 0,76 & & $61^{\circ}$ & $40^{\circ}$ & \\
\hline \multirow{3}{*}{ Pregunta 9} & I & 0,76 & $-0,62$ & 0,69 & $40^{\circ}$ & $129^{\circ}$ & $46^{\circ}$ \\
\hline & II & 0,73 & $-0,71$ & 0,23 & $43^{\circ}$ & $136^{\circ}$ & $77^{\circ}$ \\
\hline & III & 0,44 & $-0,65$ & 0,82 & $64^{\circ}$ & $131^{\circ}$ & $34^{\circ}$ \\
\hline
\end{tabular}

A partir de los resultados del análisis factorial exploratorio y del análisis de conglomerados, se comprueba la existencia de un patrón subyacente que explicite las relaciones, inter e intra, existentes entre ítems y factores. Es decir, dar respuesta empírica a la pregunta sobre el tipo de relación que, según el alumnado, se produce entre el concepto de plagio y sus elementos constitutivos, las acciones consideradas plagio que realizan dichos estudiantes y los diversos motivos de tales actividades poco éticas. Para ello, se utiliza como herramienta estadística el análisis factorial confirmatorio (AFC) con el programa Amos v21.

El diseño propuesto, que se recoge en la figura 3, consta de seis variables latentes o subyacentes que se corresponden con los factores hallados en el AFE y que a su vez son explicados por 18 variables explícitas u observadas que se corresponden con los ítems con mayor carga factorial, comentados anteriormente. Así la variable «Concepto» (C), hace referencia a los elementos constitutivos que, según el alumnado, dan cuenta del concepto de plagio y es explicada por cuatro ítems (pregunta 6):

- Copiar fragmentos de fuentes impresas (libros, enciclopedias, periódicos, artículos de revista, etc.) y añadirlos — sin citar- como partes de un trabajo propio de una asignatura.

- Hacer íntegramente un trabajo a partir de fragmentos copiados literalmente de páginas web (sin que ninguna parte del trabajo haya sido realmente escrita por el alumno/a).

- Entregar un trabajo realizado por otro alumno/a que ya había sido entregado en cursos anteriores (para la misma asignatura o para otra).

- Bajar un trabajo completo de Internet y entregarlo, sin modificar, como trabajo propio de una asignatura. 
VIOLETA CEBRIÁN-ROBLES, MANUELA RAPOSO-RIVAS, MANUEL CEBRIÁN-DE-LA-SERNA, JOSÉ ANTONIO SARMIENTO-CAMPOS

PERCEPCIÓN SOBRE EL PLAGIO ACADÉMICO DE ESTUDIANTES UNIVERSITARIOS ESPAÑOLES

La variable implícita «Parte» $(\mathrm{P})$ recoge aquellas actividades de plagio que realiza el alumnado (pregunta 8 ), pero en las que dicho plagio es parcial:

- Copiar fragmentos de fuentes impresas (libros, enciclopedias, periódicos, artículos de revista, etc.) y añadirlos — sin citar- como partes de un trabajo propio de una asignatura.

- Copiar imágenes, vídeos y sonidos de Google sin indicar la autoría $\mathrm{y}$ de dónde se obtuvo.

- Copiar de páginas web fragmentos de textos y — sin citar- pegarlos directamente en un documento - en el cual hay una parte de texto escrita por uno/a mismo/a - y entregarlo como trabajo de una asignatura.

Consecuentemente la variable «Todo»(T) da cuenta de aquellas acciones en las que el plagio es total (pregunta 8):

- Bajar un trabajo completo de Internet y entregarlo, sin modificar, como trabajo propio de una asignatura.

- Entregar un trabajo realizado por otro alumno/a que ya había sido entregado en cursos anteriores (para la misma asignatura o para otra).

- Hacer íntegramente un trabajo a partir de fragmentos copiados literalmente de páginas web (sin que ninguna parte del trabajo haya sido realmente escrita por mí).

Las otras tres variables subyacentes hacen referencia a los motivos (pregunta 9) que llevan al alumnado a realizar plagio, y que se podrían agrupar según el origen de la motivación. Cuando esta tiene su origen en motivos internos «Locus Interno» (LI):

- Escribir sobre algo que desconozco.

- Escribir sobre algo que no comprendo.

- Porque tuve que redactar un trabajo muy extenso a lo que no estoy acostumbrado/a.

Si la motivación tiene origen externo «Locus externo» (LE):

- El profesorado no tiene mucho tiempo para saber si hubo plagio.

- El profesorado no tiene muchas competencias para detectarlo.

- Porque no hay peligro, no se toma ninguna acción en los casos de plagio. 
Y cuando la motivación no existe o el origen del plagio es el «Desinterés» (D):

- Porque no se aprendía mucho en la asignatura.

- Por desinterés en la tarea.

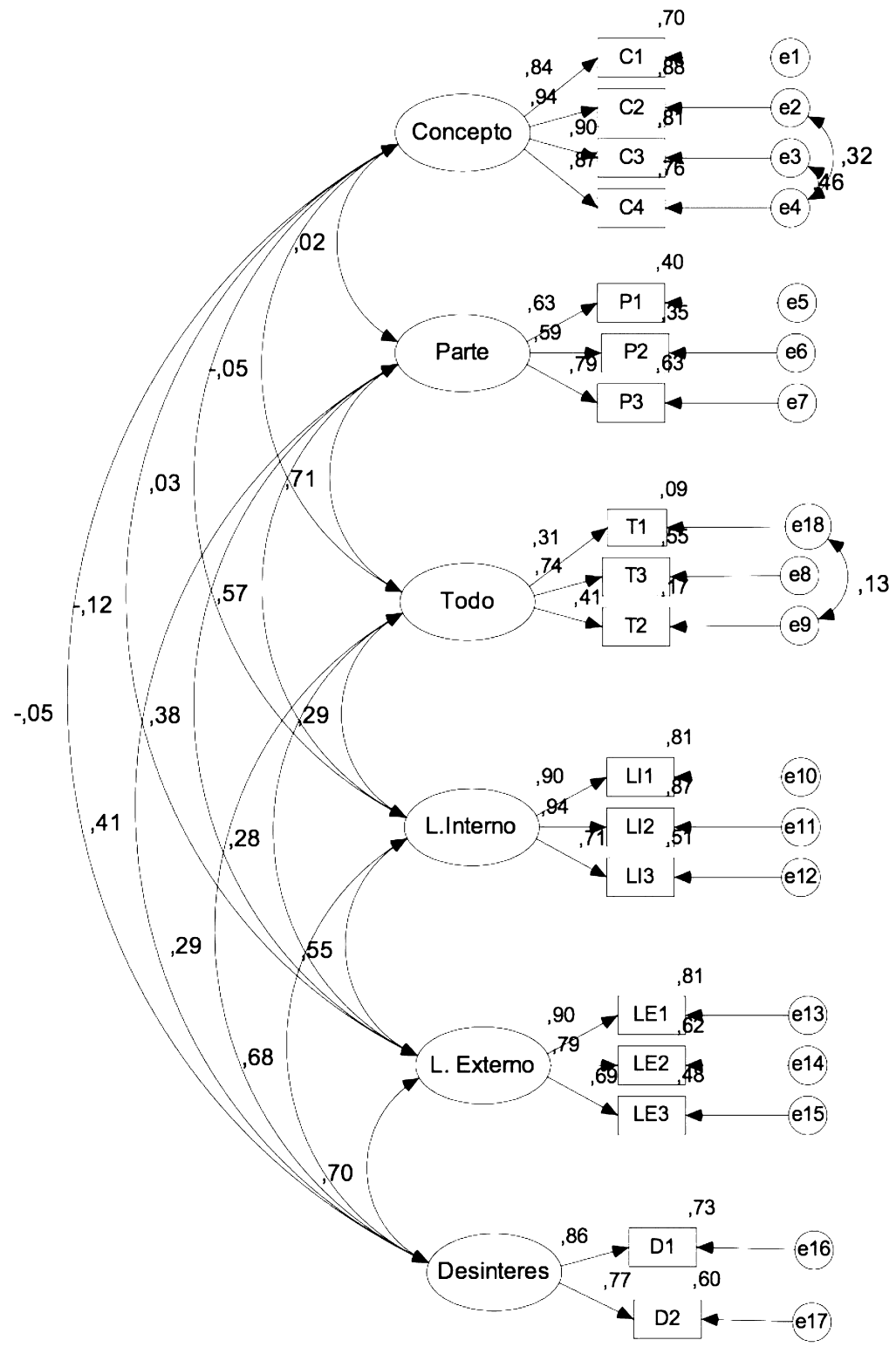

Figura 3. Patrón resultante del Análisis Factorial Confirmatorio, después de haber aplicado un Análisis Factorial Exploratorio y Cluster 
VIOLETA CEBRIÁN-ROBLES, MANUELA RAPOSO-RIVAS, MANUEL CEBRIÁN-DE-LA-SERNA, JOSÉ ANTONIO SARMIENTO-CAMPOS

PERCEPCIÓN SOBRE EL PLAGIO ACADÉMICO DE ESTUDIANTES UNIVERSITARIOS ESPAÑOLES

Los índices de la regresión son todos significativos. Los pesos más bajos, aun siendo significativos, son los correspondientes a la variable «Todo».

A través del análisis de las covarianzas se pone de manifiesto la escasa, o en este caso, nula relación entre el concepto de plagio (contenido) y las actividades de plagio que se realizan (procedimientos) como las motivaciones que dan origen a tales actividades (actitudes). En contraste con lo anterior, las relaciones entre todas las variables que dan cuenta tanto de las acciones como de las motivaciones son significativas.

Según Curran, West y Finch (1996) se establecen los límites, en valor absoluto, hasta los que se pueden considerar un comportamiento semejante al normal, en los valores comprendidos entre 2 para la asimetría y 7 para la curtosis. Cumplen el primer criterio todas las variables excepto $\mathrm{C} 1$.

Por otro lado, la literatura especializada recomienda emplear múltiples indicadores para evaluar el ajuste del modelo ( $\mathrm{Hu}, \&$ Bentler, 1998; Schreiber, et al., 2006; Ruíz, Pardo \& San Martín, 2010). Por ello, para la evaluación del ajuste global del modelo se consideraron como criterios o índices estadísticos: Chi cuadrado normalizada (CMIN/DF); índice de bondad del ajuste (GFI); índice de bondad del ajuste corregido (AGFI); índice de ajuste comparativo (CFI); error de aproximación cuadrático medio (RMSEA); y el criterio informativo de Akaike (AIC).

La relación chi cuadrado/grados de libertad es de 1,970 (condicionado por el tamaño de la muestra). El índice NFI ha de ser $>0,9$ para indicar un ajuste satisfactorio (Bentler, 1992), el valor en nuestro modelo es igual a 0,952. Los índices IFI (Bollen \& Long, 1989) y GFI (Bentler, 1992) también son superiores al valor mínimo de 0,9 para un buen ajuste (IFI $=0,976$; CFI $=0,976$ ). El criterio de información de Akaike (AIC) es de 374,45, un valor mucho menor que el modelo independiente 4879,08, lo que supone siguiendo a Ullman (1996), que el modelo presenta un buen ajuste estadístico. Por lo que respecta al índice RMSEA y para efectos de significatividad, valores que van de .05 a .08 se consideran aceptables (Hair et al., 1999). Según los índices comentados el modelo presenta un buen ajuste.

\section{CONCLUSIONES Y DISCUSIÓN}

El principal interés del trabajo reside en conocer las acciones y las motivaciones por las que los estudiantes universitarios españoles de Educación realizan prácticas deshonestas y plagian en sus trabajos. El estudio pretende comprender las ideas y las prácticas deshonestas de estos 
universitarios, especialmente porque serán futuros docentes al mismo tiempo que modelo y factor determinante de las prácticas de su alumnado. Para ello, se desarrolla un modelo factorial que permite identificar el concepto que poseen sobre el constructo "plagio», las acciones en que realizan plagio «parcial» $\mathrm{y}$ «total», así como los motivos para plagiar: «internos» (algo que desconozco, no comprendo...), «externos» (falta tiempo, competencias...) y el «desinterés» o ausencia de motivación por la materia o la tarea planteada.

Así, podemos afirmar coincidiendo con otros estudios desarrollados en contextos diferentes (Lau, Yuen, \& Park, 2013), que existe un patrón subyacente en los estudiantes universitarios españoles de educación, que muestra la relación existente entre conocimiento y práctica deshonesta. Desde los resultados obtenidos hallamos un valor en el modelo de 0,952 $\mathrm{y}$ un alpha total de 0,881 . Valores que sostienen los datos descriptivos que observamos en las 18 variables con mayor carga factorial, y que resumimos aquí en los tres puntos más relevantes:

a) Los estudiantes disponen de una definición clara sobre el plagio, como la "copia y utilización de un fragmento de forma integra o parcial presentada como un trabajo propio", aunque no incluyen el autoplagio ni la apropiación de material audiovisual (figura 2). En el estudio de Fish \& Hura, (2013) encontraron que los estudiantes perciben que sus compañeros plagian en un promedio de 3,5 (escala de 1 a 6), ello puede ser un síntoma de que se sienten más amenazados a plagiar, explicable sobre todo por la influencia social que el grupo tiene sobre la personalidad de los jóvenes.

b) Los motivos internos (locus interno) que utilizan los estudiantes para justificar el plagio son el desconocimiento, la incomprensión y la poca práctica en la redacción de trabajos extensos. Resultados que nos señalan la necesidad de mayor acompañamiento y formación desde la institución, dado que los estudiantes sostienen que el plagio disminuye a medida que son acompañados por los docentes y existe una formación específica (Ochoa \& Cueva, 2016).

c) El riesgo a ser detectados es uno de los tres constructos del modelo Lau, Yuen, \& Park (2013). En nuestro estudio, los motivos externos (locus externo) están motivados principalmente por la imposibilidad del docente de detectar el plagio por falta de tiempo, la falta de competencia del docente y la inexistencia de peligro si eres detectado. Sin duda, también existen otras razones asociadas a la organización curricular y la falta de formación y orientación a los estudiantes, como de prevención por parte de los docentes e instituciones (Comas-Forgas \& Sureda-Negre, 2010), como también las creencias, actitudes y personalidad de los estudiantes (Kuntz \& Butler, 2014). 
VIOLETA CEBRIÁN-ROBLES, MANUELA RAPOSO-RIVAS, MANUEL CEBRIÁN-DE-LA-SERNA, JOSÉ ANTONIO SARMIENTO-CAMPOS

PERCEPCIÓN SOBRE EL PLAGIO ACADÉMICO DE ESTUDIANTES UNIVERSITARIOS ESPAÑOLES

Estas tres conclusiones permiten dibujar un patrón (ver figura 3) en el que, a pesar de conocer (concepto) lo que es el plagio y sus consecuencias, el propio hecho de plagiar se justifica por, al menos, tres tipos de causas interrelacionadas: internas (propias del sujeto, con un locus interno), externas (ajenas al sujeto, con un locus externo) y el desinterés. La relación de cada una de estas causas con el plagio es diferente, así el alumnado de manera mayoritaria procura que el plagio cometido sea parcial, especialmente en aquellos casos en los que la motivación a cometerlo deriva de causas internas relacionadas con la falta de pericia en el tema y la tarea.

Desde estos resultados cabe pensar, de acuerdo con Cavanillas (2008), que la alusión al carácter negativo del plagio siempre resulta instructiva y evitará que el estudiante se ampare en una supuesta ignorancia; por lo que se recomienda la incorporación a los programas de las asignaturas de algún criterio de evaluación que elimine, prevenga o reduzca la puntuación de los trabajos plagiados. Al mismo tiempo, acciones que fomenten un ambiente más favorable a las buenas prácticas individuales e institucionales, como demostraron Gullifer \& Tyson (2014) en una investigación con 3.405 estudiantes universitarios, donde solo la mitad de los participantes había leído la política sobre el plagio y la integridad académica, al mismo tiempo que era evidente cierta confusión con respecto a qué conducta constituye plagio. Es urgente que los estudiantes comprendan la necesidad y trascendencia de sus tareas, es necesario que busquen sentido al trabajo académico desde el inicio de la carrera, y si es anterior mejor, haciendo un esfuerzo por entender lo que se está haciendo, con qué y para qué (Ramírez-Barreto, 2017).

Esto es así, cuando observamos en la literatura cómo la utilización de plataformas tecnológicas para prevenir el plagio no es suficiente, y que existen una multiplicación de factores. No obstante, y cuando se considera como una medida más y los estudiantes conocen de su existencia, sí posee un efecto en la prevención (Youmans, 2011). Al mismo tiempo, crecen las prácticas educativas en nuevos escenarios como las redes sociales, por lo que se necesitan sofisticadas respuestas tecnológicas con "patrones avanzados de plagio» (Zrnec \& Lavbič, 2017), así como nueva gestión y uso proactivo de las mismas. Como complemento a las tecnologías, también se necesitan acciones encaminadas a un cambio en las prácticas de los docentes y los servicios institucionales tanto en los niveles universitarios como los no universitarios (Chang, 2009), que nos permitan analizar cómo funcionan las estrategias de prevención, y comprender todas las dimensiones que entran en juego en el aprendizaje de los estudiantes a la hora de presentar sus evidencias. Esto nos exige comprender mejor y seguir investigando sobre qué piensan los estudiantes y cómo justifican sus prácticas. 


\section{NOTAS}

1 Proyecto $\mathrm{I}+\mathrm{D}+\mathrm{i}$ titulado Estudio del impacto de las e-rúbricas federadas en la evaluación de las competencias en el Practicum (2014-2017). Financiado por la convocatoria de Excelencia del Ministerio de Economía y Competitividad, n. ${ }^{\circ}$ EDU2103-41974-P. 
VIOLETA CEBRIÁN-ROBLES, MANUELA RAPOSO-RIVAS, MANUEL CEBRIÁN-DE-LA-SERNA, JOSÉ ANTONIO SARMIENTO-CAMPOS

PERCEPCIÓN SOBRE EL PLAGIO ACADÉMICO DE ESTUDIANTES UNIVERSITARIOS ESPAÑOLES

\section{REFERENCIAS BIBLIOGRÁFICAS}

Adam, L., Anderson, V. \& SpronkenSmith, R. (2017). 'It's not fair': Policy discourses and students' understandings of plagiarism in a New Zealand university. Higher Education, 74(1), 17-32. 10.1007/s10734-0160025-9

Adam, L. (2016). Student perspectives on plagiarism. In T. Bretag (Ed.). (2016). Handbook of academic integrity. (pp.519-533). Singapore: Springer.

Amiri, F., \& Razmjoo, S. (2016). On iranian EFL undergraduate students' perceptions of plagiarism. Journal of Academic Ethics, 14(2), 115-131. 10.1007/s10805-015-9245-3

Balbuena, S.E., \& Lamela, R.A. (2015). Prevalence, motives and views of academic dishonesty in Higher Education. Asia Pacific Journal of Multidisciplinary Research, 3(2), 69-75.

Batista_Foguet, J.M. y Coenders, G. (2000). Modelos de ecuaciones estructurales. Madrid: La Muralla.

Bentler, P.M. (1992). On the fit of models to covariances and methodology to the Bulletin. Psychological bulletin, 112(3), 400 .

Bretag, T. (Ed.). (2016). Handbook of academic integrity. Singapore: Springer.

Bollen, K.A. \& Long, J.S. [Eds.] (1989) Testing structural equation models. Newbury Park, CA: Sage.

Caldevilla Domínguez, D. (2010). Internet como fuente de información para el alumnado universitario. Cuadernos de Documentación Multimedia, 21, 141-157. Recuperado de: https://goo.gl/CtEqn6

Cavanillas, S. (2008). El ciberplagio en la normativa universitaria. En:
R. Comas-Forgas \& J. Sureda-Negre (coords.). (2008). El ciberplagi acadèmic [dossier en línia]. Digithum, 10. UOC. Recuperado de: http://goo.gl/ $7 \mathrm{XqONt}$

Cebrián-Robles, V., Raposo-Rivas, M., \& Sarmiento-Campos, J.A. (2016). ¿Ética o prácticas deshonestas? el plagio en las titulaciones de educación .Revista de Educación, 374, 161-186. 10.4438/1988-592X-RE-2016-374-330

Chang, S. (2009). El derecho de autor en el aula- Mío, tuyo, suyo. Revista de la OMPI, 1. Recuperado de: https://goo. $\mathrm{gl} / \mathrm{XxJjtN}$

Chankova, M. (2017). Dealing with Students' Plagiarism Pre-Emptively Through Teaching Proper Information Exploitation. International Journal for the Scholarship of Teaching and Learning, 11(2), 4.

Comas-Forgas, R., \& Sureda-Negre, J., (2010). Academic Plagiarism: Explanatory Factors from Students' Perspective. J. Acad Ethics, 8, 217-232. 10.1007/s10805-010-9121-0

Comas-Forgas, R., \& Sureda-Negre, J. (2007). Ciber-plagio académico. Una aproximación al estado de los conocimientos. Revista Textos de la CiberSociedad, 10. Recuperado de: https://goo.gl/FegNp2.

Cosma, G., Joy, M., Sinclair, J., Andreou, M., Zhang, D., Cook, B., \& Boyatt, R. (2017). Perceptual comparison of source-code plagiarism within students from UK, China, and South Cyprus higher education institutions. ACM Transactions on Computing Education (TOCE), 17(2), 8.

Curran, P.J., West, S.G. \& Finch, J.F. (1996). The robustness of test statistics 
to nonnormality and specification error in confirmatory factor analysis. Psychological Methods 1, 16-29.

Dalal, N. (2015). Responding to plagiarism using reflective means. International Journal for Educational Integrity, 11(1), 4. 10.1007/s40979-015-0002-6

Díaz-Emparanza, I. (1996). Selecting the Number of Replications in a Simulation Study. Working Paper 1996-1. Recuperado de: https://goo.gl/WNB2dr. http://dx.doi.org/10.2139/ssrn.1582

Do Ba, K.; Do Ba, K.; Lam, Q.D.; Le, Dao Thanh Binh An; Nguyen, P.L.; Nguyen, P.Q. \& Pham, Q.L. (2017). Student plagiarism in higher education in Vietnam: An empirical study. Higher Education Research \& Development, 36(5), 934-13. 10.1080/07294360.2016.1263829

Eaton, S., Guglielmin, M., \& Otoo, B. (2017). Plagio: Pasar de enfoques punitivos a proactivos. En A.P. Preciado Babb, L. Yeworiew \& S. Sabbaghan (Eds.). Actas Seleccionadas de la Conferencia IDEAS 2017: Liderando la Conferencia sobre Cambio Educativo (pp. 28-36). Calgary, Canadá: Werklund School of Education, Universidad de Calgary.

Efron, B., \& Tibshirani, R.J. (1994). An Introduction to the Bootstrap. Boca Ratón, Flo.: CRC Press.

Ehrich, J., Howard, S. J., Mu, C., \& Bokosmaty, S. (2016). A comparison of Chinese and Australian university students' attitudes towards plagiarism. Studies in Higher Education, 41(2), 231246. 10.1080/03075079.2014.927850

Ennam, A. (2017). Systematic Analysis of the Effects of Digital Plagiarism on Scientific Research: Investigating the Moroccan Context--Ibn Tofail University as Case Study. Journal of Education and Practice, 8(2), 133-141.
Fish, R., \& Hura, G. (2013). Students' perceptions of plagiarism. Journal of the Scholarship of Teaching and Learning, 13(5), 33-45.

Guerrero, P., Mercado, J. \& Marina, L. (2017). La deshonestidad, elemento que altera la integridad en las prácticas académicas en las Instituciones de Educación Superior. Estudios de caso comparados. Investigación y formación pedagógica. Revista del Ciegc. O(5), 6-25. Recuperado de: https:/goo.gl/qTbHAi

Guerrero, P., Marina, L. \& Mercado, J. (2016). Los indicadores de calidad y su relación con la ética en la producción académica. Estudio de casos comparados. RIDE Revista Iberoamericana para la Investigación y el Desarrollo Educativo, 7(13), 197-219.

Gullifer, J.M. \& Tyson, G.A. (2014). Who has read the policy on plagiarism? Unpacking students'understanding of plagiarism, Studies in Higher Education, 39(7), 1202-1218, 10.1080/03075079.2013-777412

Hair, J., Anderson, R., Tatham, R., \& Black, W. (1999). Análisis Multivariante. Madrid: Prentice Hall.

Hall, R.J., Snell, A.F., \& Singer Foust, M., (1999). Item parceling strategies in SEM: Investigating the subíle effects of unmodeled secondary constructs. Organizational Research Methods, 2, 233-256.

Hayes, N., \& Introna, L.D. (2005). Cultural values, plagiarism, and fairness: When plagiarism gets in the way of learning. Ethics \& Behavior, 15(3), 213-231. 10.1207/ s15327019eb1503_2

Heckler, N.C., Forde, D.R., \& Bryan, C.H. (2013). Using writing assignment designs to mitigate plagiarism. Teaching Sociology, 41(1), 94-105. 10.1177/0092055X12461471 
VIOLETA CEBRIÁN-ROBLES, MANUELA RAPOSO-RIVAS, MANUEL CEBRIÁN-DE-LA-SERNA, JOSÉ ANTONIO SARMIENTO-CAMPOS

PERCEPCIÓN SOBRE EL PLAGIO ACADÉMICO DE ESTUDIANTES UNIVERSITARIOS ESPAÑOLES

Hernández, R., Fernández, C. \& Baptista, P. (2014). Metodología de la investigación. México: McGrawHill.

Huh, J., Delorme, D.E., \& Reid, L.N. (2006), Perceived Third-Person Effects and Consumer Attitudes on Prevetting and Banning DTC Advertising. Journal of Consumer Affairs, 40, 90-116.

Hu, L.T., \& Bentler, P.M.,(1998). Fit indices in covariance structure modeling: Sensitivity to underparameterized model misspecification. Psychological Methods, 3, 424-453.

Hu, G., \& Sun, X. (2017). Institutional policies on plagiarism: The case of eight Chinese universities of foreign languages/international studies. System, 66, 56-68. Recuperado de: https://goo.gl/Ne3qZk

Kerlinger, F. \& Lee, H. (2002). Investigación del comportamiento. México: McGrawHill/ Interamericana.

Khoshsaligheh, M., Mehdizadkhani, M., \& Keyvan, S. (2017). Severity of Types of Violations of Research Ethics: Perception of Iranian Master's Students of Translation. Journal of Academic Ethics, 15(2), 125-140.

Kokkinaki, A., Demoliou, C., \& Iakovidou, M. (2015). Students' perceptions of plagiarism and relevant policies in Cyprus. International Journal for Educational Integrity, 11(1), 1-11. 10.1007/s40979-015-0001-7

Kuntz, J.R. C., \& Butler, C. (2014). Exploring individual and contextual antecedents of attitudes toward the acceptability of cheating and plagiarism. Ethics \& Behavior, 24(6), 478-494. $10.1080 / 10508422.2014 .908380$

Lau, G.K.K., Yuen, A.H.K., \& Park, J. (2013). Toward an analytical model of ethical decision making in plagiarism.
Ethics \& Behavior, 23(5), 360-377. $10.1080 / 10508422.2013 .787360$

Lloret-Segura, S., Ferreres-Traver, A., Hernández-Baeza, A., y TomásMarco, I. (2014). El análisis factorial exploratorio de los ítems: una guía práctica, revisada y actualizada. Anales de Psicología, 30(3). https://doi. org/10.6018/analesps.30.3.199361

López, J., (2014). Analyzing and reducing plagiarism at university. European Journal of Education and Psychology, 7(2), 131-140.

McGowan, S., \& Lightbody, M. (2008). Enhancing students' understanding of plagiarism within a discipline context. Accounting Education, 17(3), 273-290. 10.1080/09639280701612168

Montecinos, A. (2013). Plagio y ética de la investigación científica/Plagiarism and ethics of scientific research. Revista Chilena De Derecho, 40(2), 711-726. Recuperado de: https://goo. gl/Tch8Vf

Ochoa, L. \& Cueva, A (2016). Percepciones de estudiantes acerca del plagio. Encuentros, 14 (2), pp.25-41. Recuperado de: https://goo.gl/qgwSkV

Park, C. (2003). In other (people's) words: Plagiarism by university students-literature and lessons. Assessment \& Evaluation in Higher Education, 28(5), 471-488. 10.1080/02602930301677

Perelló, S.(2009). Metodología de la Investigación Social. Madrid: Dykinson S.L.

Ramírez-Barreto, A.C. (2017). El plagio académico. experiencias y algunas ideas para desalentarlo de manera más efectiva. Ciencia Nicolaita, 70, 7-22. Recuperado de: https://goo.gl/ c5LwQM 
Real Academia Española. (2001). Diccionario de la lengua española (23. ${ }^{\mathrm{a}}$ ed.). Madrid: RAE.

Revelle, W. (2017). Psych: Procedures for Personality and Psychological Research. Evanston, Ill: Northwestern University. Recuperado de https://goo.gl/oa2Cr8

Revelle, W. \& Zinbarg, R.E. (2009) Coefficients alpha, beta, omega and the glb: comments on Sijtsma. Psychometrika, 74(1), 145-154.

Revelle, W. \& Rocklin, T. (1979). Very Simple Structure: an Alternative Procedure for Estimating the Optimal Number of Interpretable Factors, Multivariate Behavioral Research, 14, 403-414.

Revelle, W. (1979). Hierarchical clusteranalysis and the internal structure of tests. Multivariate Behavioral Research, 14(1), 57-74.

Ruíz, M., Pardo, A., \& San Martín, R. (2010). Modelos de ecuaciones estructurales. Papeles del psicólogo, 31(1), 34-45.

Ruipérez, G., \& García-Cabrero, J.C. (2016). Plagio e integridad académica en Alemania. Comunicar: Revista Científica de Comunicación y Educación, 24(48), 9-17. https://doi. org/10.3916/C48-2016-01

Sanvicén Torné, P., \& Molina Luque, F. (2015). Efectos del uso de internet como fuente principal de información. Evidencias en estudiantes de primer curso universitario. Prisma Social, (15). Recuperado de: https://goo.gl/ zvUtTt

Sattler, S., Wiegel, C., \& Veen, F.V. (2017). The use frequency of 10 different methods for preventing and detecting academic dishonesty and the factors influencing their use. Studies in Higher Education, 42(6), 1126-1144.
Sattler, S., Graeff, P., \& Willem, S (2013). Explaining the decision to plagiarize: An empirical test of the interplay between rationality, norms, and opportunity. Deviant Behavior, 34(6), 444-463. 10.1080/01639625.2012.735909

Schreiber, J. B., Nora, A., Stage, F.K., Barlow, E.A., \& King, J. (2006). Reporting structural equation modeling and confirmatory factor analysis results: A review. The Journal of educational research, 99(6), 323-338.

Sureda-Negre, J., Comas-Forgas, R., \& Oliver-Trobat, M.-F. (2015). Plagio académico entre alumnado de secundaria y bachillerato: Diferencias en cuanto al género y la procrastinación. Comunicar, 22(44), 103-111. http://doi. org/10.3916/C44-2015-11

Sureda-Negre, J., Comas-Forgas, R., \& Morey, M. (2009). Las causas del plagio académico entre el alumnado universitario según el profesorado. Revista iberoamericana de educación, 50, 197-220.

Tayan, B.M. (2016). Academic Misconduct: An Investigation into Male Students' Perceptions, Experiences \& Attitudes towards Cheating and Plagiarism in a Middle Eastern University Context. Journal of Education and Learning, 6(1), 158.

Tirado, R., \& Aguaded, J. I. (2012). Influencia de las medidas institucionales y de la competencia tecnológica sobre la docencia universitaria a través de plataformas digitales. Relieve, 18(1). Recuperado de: https://goo.gl/5e9BNT

Ullman, J.D. (1996). Structural Equation Modellin. In B.G. Tabachnick \& L.S. Fidell (eds.) Using multivariate statistics (pp. 709-812). Nueva York: Harper-Collins College Publishers. 
Velicer, W. (1976) Determining the number of components from the matrix of partial correlations. Psychometrika, 41, 321-327.

Youmans, R. J. (2011). Does the adoption of plagiarism-detection software in higher education reduce plagiarism? Studies in Higher Education, 36(7), 749761. 10.1080/03075079.2010.523457
Zarfsaz, E., \& Ahmadi, R. (2017). Investigating Some Main Causes and Reasons of Writing Plagiarism in an EFL Context. International Journal of Applied Linguistics and English Literature, 6(5), 214-223.

Zrnec, A., \& Lavbič, D. (2017). Social network aided plagiarism detection. British Journal of Educational Technology, 48(1), 113-128. 10.1111/ bjet.12345 


\section{PERFIL ACADÉMICO Y PROFESIONAL DE LOS AUTORES}

Violeta Cebrián-Robles. Doctoranda en el programa de Doctorado Interuniversitario en Equidad e Innovación en Educación de la Universidad de Vigo. Licenciada en Derecho. Máster en Educación. Máster en Derecho. Experto en Mediación. Gestión de proyectos y Tutora de acciones formativas online en empresas del sector de formación. Miembro del Grupo de investigación en Equidad e Innovación en Educación de la Universidad de Vigo. Líneas: Educación Superior, Recursos Educativos Abiertos, derechos de autor y formación e-learning. Orcid: http://orcid. org/0000-0002-6862-8270

Manuela Raposo-Rivas. Profesora Titular de Nuevas Tecnologías aplicadas a la Educación. Docencia e investigación relacionada con la formación de futuros docentes, la tecnología educativa, las prácticas preprofesionales y la innovación en educación. Sobre esta temática posee publicaciones y participa en diferentes redes y proyectos de investigación nacionales, autonómicos e internacionales. Pertenece a Comités Científicos y de Redacción de diferentes revistas indexadas en el ámbito de la Educación y las TIC. Orcid: http://orcid.org/0000-0001-7781-7818

Manuel Cebrián-de-la-Serna. Catedrático de Tecnología Educativa. Líneas: a) Innovación educativa vs tecnológica; b) Enseñanza universitaria; c) Prácticum. 10 años como director de distintos servicios de formación para docentes - 1994-2003- (ICE; Innovación educativa y Servicio de Enseñanza Virtual). Director del grupo investigación Gtea, Junta de Andalucía (SEJ462). Ha dirigido diferentes proyectos $\mathrm{I}+\mathrm{D}+\mathrm{i}$ con socios de universidades en Asia Central, Europa, EEUU y Latinoamerica (erubrica.org). Editor Revistapracticum.com. Web Personal: http://gtea.uma.es/mcebrian. orcid. org/0000-0002-0246-7398

José Antonio Sarmiento-Campos. Doctor en Psicopedagogía. Profesor de Enseñanza Secundaria, especialidad de orientación educativa, y profesor asociado en el Departamento de Didáctica, Organización Escolar y Métodos de Investigación de la Universidad de Vigo. Master en técnicas actuales de estadística aplicada. Sus trabajos se centran en las áreas de la orientación psicopedagógica, diversidad en la etapa de educación infantil y formación del profesorado. Miembro de la Asociación Interuniversitaria de Investigación Pedagógica (AIDIPE). Orcid: https://orcid.org/0000-0003-3537-1197

Dirección de los autores: Violeta Cebrián-Robles

Manuela Raposo-Rivas

Dpto. Didáctica, Organización Escolar y Métodos de Investigación 
VIOLETA CEBRIÁN-ROBLES, MANUELA RAPOSO-RIVAS, MANUEL CEBRIÁN-DE-LA-SERNA,

PERCEPCIÓN SOBRE EL PLAGIO ACADÉMICO DE ESTUDIANTES UNIVERSITARIOS ESPAÑOLES

Facultad de Ciencias de la Educación

Despacho 20. Edificio 2

Universidad de Vigo

Campus As Lagoas, s/n

32004 Ourense (España)

E-mail: violetacbr@uvigo.es

mraposo@uvigo.es

Manuel Cebrián de-la-Serna

Facultad de Educación

Universidad de Málaga

Campus Teatinos

Bulevar Louis Pasteur, 25

Málaga (España) D.P. 29071

E-mail: mcebrian@uma.es

José Antonio Sarmiento-Campos

Facultad de Ciencias de la Educación

Universidad de Vigo

Campus As Lagoas, s/n

Pabellón n. ${ }^{\circ} 1-3$. $^{\text {a }}$ Planta Despacho n. ${ }^{\circ} 4$

Ourense (España) D.P. 32004

E-mail: sarmiento@uvigo.es

Fecha Recepción del Artículo: 15. Octubre. 2017

Fecha Modificación del Artículo: 04. Febrero. 2018

Fecha Aceptación del Artículo: 10. Febrero. 2018

Fecha Revisión para Publicación: 22. Febrero. 2018 